\title{
Concentración de nutrientes en hoja y calidad de pepino en plantas injertadas bajo condiciones salinas
}

\author{
Concentration of nutrients in leaf and cucumber quality in plants \\ grafted under salin conditions \\ Onécimo Grimaldo-Juárez ${ }^{1}$, Ángel Manuel Suárez-Hernández²*, \\ Emmanuel Alejandro Vargas-Hernández ${ }^{2}$, Laura Dennise Carrazco-Peña ${ }^{2}$, \\ Luis Alberto Morales-Zamorano ${ }^{2}$
}

\section{RESUMEN}

La salinidad es uno de los principales problemas en los sistemas de producción de pepino (Cucumis sativus) en zonas áridas y semiáridas, afectando la homeostasis de iones y consecuentemente la calidad de la fruta. Una alternativa de producción es el uso del injerto, sin embargo, el efecto depende de la combinación específica portainjerto-variedad. Esta investigación tuvo como objetivo evaluar la concentración de iones en hoja y calidad de fruto en pepino variedad Tirano en dos condiciones de planta (normal e injertada) bajo tres niveles de salinidad (3,0; 4,5 y 6,0 dS m-1). El contenido de potasio y la relación potasio-sodio en hoja fueron alterados por la combinación condición de planta y nivel de salinidad, expresando mayor valor en planta injertada a conductividad de 3,0 dS m${ }^{-1}$. La concentración de sodio en hoja se incrementó con el nivel de salinidad. En condición de planta, la concentración de sodio fue más elevada en normal que en injertada. El peso y diámetro de fruto variaron con la condición de planta y nivel de salinidad. La planta injertada a salinidad de $6,0 \mathrm{dS} \mathrm{m}^{-1}$ registró mayor peso y diámetro de fruto. La longitud, firmeza externa e interna y el contenido de sólidos solubles oscilaron entre 16,6 y $19,2 \mathrm{~cm} ; 73,8$ y $74,1 \mathrm{~N} ; 58,0$ y $74,1 \mathrm{~N} ;$ y 4,9 y $5,1 \%$, respectivamente, sin efectos significativos por los factores evaluados. El portainjerto Forticuke F1 favorece la asimilación de potasio y relación de potasio/sodio, reduce el contenido de sodio en hoja a concentración de 3,0 y 4,5 dS m ${ }^{-1}$ e incrementa el peso y diámetro de fruto en pepino en ambiente de $6,0 \mathrm{dS} \mathrm{m}^{-1}$. La longitud, firmeza y contenido de sólidos solubles no fueron modificados por el portainjerto en los distintos niveles de salinidad.

Palabras clave: Cucumis sativus, injerto, salinidad, concentración de iones, calidad de fruto.

\begin{abstract}
Salinity is one of the main problems in cucumber production systems (Cucumis sativus) in arid and semi-arid areas, affecting ion homeostasis and consequently fruit quality. An alternative of production is the use of graft, however, the effect depends on a specific rootstock-variety combination. Therefore, in the present investigation, the objective was to evaluate the concentration of ions in leaf and fruit quality in cucumber variety Tirano in two plant conditions (normal and grafted) under three levels of salinity (3.0,

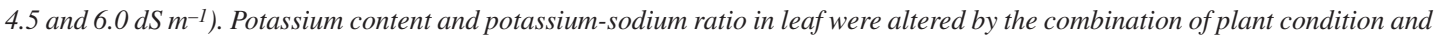
salinity level, expressing higher value in the grafted plant at a conductivity of $3.0 \mathrm{dS} \mathrm{m}^{-1}$. Sodium concentration in leaf increased with the level of salinity. In plant conditions, sodium concentration was higher in normal than ingrafted. Fruit weight and diameter varied with plant conditions and salinity levels. The grafted plant at a salinity of $6.0 \mathrm{dS} \mathrm{m}^{-1}$ registered higher weight and diameter of fruit. Length, external and internal firmness and soluble solids content ranged from 16.6 to $19.2 \mathrm{~cm}, 73.8$ to $74.1 \mathrm{~N}, 58.0$ to $74.1 \mathrm{~N}$ and 4.9 to $5.1 \%$, respectively, without significant effects by the factors evaluated. The Forticuke F1 rootstock favors the assimilation of potassium and potassium/sodium ratio, reduces the sodium content in leaf at a concentration of 3.0 and $4.5 \mathrm{dS} \mathrm{m} \mathrm{m}^{-1}$

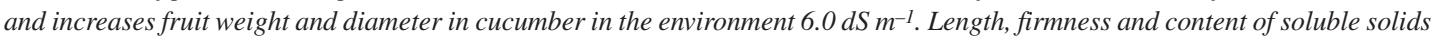
were not modified by the rootstock at different levels of salinity.
\end{abstract}

Keywords: Cucumis sativus, graft, salinity, concentration of ions, fruit quality.

1 Universidad Autónoma de Baja California, Instituto de Ciencias Agrícolas, Ejido Nuevo León, Mexicali, Baja California, México.

2 Universidad Autónoma de Baja California, Facultad de Ingeniería y Negocios San Quintín, Ejido Padre Kino, Ensenada, Baja California, México.

* Autor para correspondencia: suarez.angel@uabc.edu.mx

Fecha de Recepción: 4 de Marzo, 2020.

Fecha de Aceptación: 28 de Enero, 2020. 


\section{Introducción}

La salinidad de los suelos es uno de los principales problemas en todo el mundo. En zonas áridas y semiáridas los problemas de salinidad se incrementan por efecto de alta temperatura, disminución pluvial y aumento de concentración salina en agua de riego (Pulido-Madrigal, 2016). El impacto de la salinidad es directamente en la producción de los cultivos. La presencia de cloruro de sodio induce disminución en contenido relativo de agua y minerales como potasio, calcio y proporción potasio/sodio en plantas de pepino, así como aumento en la concentración de sodio en el tejido y, consecuentemente, estrés oxidativo (Mohsin et al., 2019).

La reducción de clorofila, tasa de asimilación neta de $\mathrm{CO}_{2}$, conductancia estomática, decaimiento no fotoquímico y eficiencia fotoquímica del PSII son otros de los efectos del estrés salino (Elsehery et al., 2020), lo que se refleja en una menor biomasa y producción en plantas de pepino. El rendimiento decrece en 5,7\% por cada incremento de $1 \mathrm{dS} \mathrm{m}^{-1}$ de CE en el agua de riego, principalmente debido a la reducción del número de frutos (Wan et al., 2010). La firmeza y acidez del fruto no se modifican por efecto de la salinidad (Colla et al., 2013).

Una de las estrategias para contrarrestar los problemas de salinidad es el empleo de la técnica del injerto. Eel injerto en plantas de pepino irrigadas con agua salina ha demostrado tener menor efecto negativo sobre el peso seco y el área foliar de la variedad (Huang et al., 2010). Limita la absorción y transporte de $\mathrm{Na}^{+}$, favorece la absorción de $\mathrm{K}^{+} \mathrm{y}$ relación de $\mathrm{K}^{+} / \mathrm{Na}^{+}$(Zhu et al., 2008). No obstante, la concentración de $\mathrm{K}^{+}, \mathrm{Na}^{+}$y la relación $\mathrm{K}^{+} / \mathrm{Na}^{+}$en el follaje de plantas injertadas de pepino pueden ser favorables o nulas al emplear híbridos de calabaza (C. máxima x C. moschata) bajo concentración salina (Usanmaz y Abak, 2019).

Plantas injertadas de pepino con el portainjerto Chilacayote (Cucurbita ficifolia) bajo estrés de $\mathrm{NaCl}$ han presentado mayor producción y contenido de azúcar soluble, sin alterar el peso del fruto (Huang et al., 2009). El peso del fruto es afectado por los portainjertos Affyne y P360 (C. maxima $x$ C. moschata), mientras que la firmeza y el contenido de sólidos solubles no son modificados (Colla et al., 2013). Esto indica que la calidad de la fruta dependerá de la combinación portainjerto-variedad. Por lo anterior, en esta investigación se evaluó la concentración de iones en hoja y calidad de fruto en pepino (Cucumis sativus) variedad Tirano injertado sobre el portainjerto Forticuke F1 bajo tres niveles de salinidad.

\section{Materiales y métodos}

El estudio se llevó a cabo en el ciclo primaveraverano del año 2019, en un invernadero de baja tecnología cubierto con lámina de policarbonato del Instituto de Ciencias Agrícolas de la Universidad Autónoma de Baja California, Mexicali, Baja California. La ubicación geográfica es $32^{\circ} 24^{\prime} 19^{\prime}$ " latitud norte y $115^{\circ} 11^{\prime} 48^{\prime \prime}$ longitud oeste, con una altitud de $10 \mathrm{msnm}$. Las plántulas se colocaron en bolsas de polietileno negro calibre 600 de $35 \mathrm{x}$ $35 \mathrm{~cm}$ (capacidad de $10 \mathrm{~L}$ ), que contenían una mezcla de sustrato peat moss y arena en una relación 1:3.

El material genético utilizado como vástago fue pepino de tipo americano variedad Tirano (Enza zaden, México) y el genotipo empleado como portainjerto fue el híbrido (C. maxima $\mathrm{x}$ C. moschata) Forticuke F1 (Nunhems, México). El injerto se realizó cuando el portainjerto y la variedad presentaron la primera hoja verdadera. El tipo de injerto utilizado fue de púa de acuerdo a la metodología de Maroto et al., (2002). Las plántulas recién injertadas se mantuvieron en una cámara ambiental con humedad relativa entre $85 \pm 5 \%$ y temperatura de $22,5 \pm 2,5^{\circ} \mathrm{C}$ durante un período de $6 \mathrm{~d}$. El proceso de aclimatización se inició al séptimo día, y consistió en bajar gradualmente la humedad relativa en $70 \pm 10 \%$ por un período de 4 d. Las plántulas injertadas se mantuvieron en el invernadero por un período de $14 \mathrm{~d}$ previo al trasplante en macetas.

Las plántulas normales e injertadas fueron trasplantadas a los $30 \mathrm{~d}$ posteriores al injerto en bolsas de polietileno perforadas para facilitar el drenaje. Los riegos se aplicaron con agua de $1,1 \mathrm{dS} \mathrm{m}^{-1}$ y un $\mathrm{pH}$ de 8.4 , en tres lapsos durante el día y un volumen de $250 \mathrm{ml} /$ maceta. A los 14 días posteriores a la plantación se efectuaron los riegos con solución nutritiva (en meq $\mathrm{L}^{-1}$ ) de 8,00 $\mathrm{K}^{+} ; 8,00 \mathrm{Ca}^{+2} ; 2,70 \mathrm{Mg}^{+2} ; 1,25 \mathrm{NH}_{4}^{+} ; 16,00 \mathrm{NO}_{3}^{-}$; 2,70 $\mathrm{SO}_{4}^{-2}$ y $1,25 \mathrm{H}_{2} \mathrm{PO}_{4}^{-}$. Los micronutrientes fueron aportados con $15 \mathrm{mg} \mathrm{L}^{-1}$ de fertilizante Haifa Micro Comb. El manejo cultural de las plantas fue acorde a los sistemas de producción de los agricultores de la región, con un sistema de conducción a un solo tallo. 
El experimento se realizó con un arreglo factorial $2 \times 3$ en un diseño de bloques al azar con cuatro repeticiones y unidad experimental de 15 plantas. Los tratamientos evaluados resultaron de la combinación condición de la planta $(\mathrm{P})$ y nivel de salinidad (S). Las condiciones de la planta fueron normal e injertada de la variedad de pepino Tirano. Los niveles de salinidad fueron 3,$0 ; 4,5$; y $6,0 \mathrm{dS} \mathrm{m}^{-1}$, los que se obtuvieron al añadir 1,5 ; 15,0 y $30,0 \mathrm{mM}$ de $\mathrm{NaCl}$, respectivamente, a la solución nutritiva.

Las variables evaluadas fueron el contenido de $\mathrm{NO}_{3}{ }^{-}$(ppm), $\mathrm{K}^{+}$(ppm), $\mathrm{Na}^{+}$(ppm), y relación $\mathrm{K}^{+}$/ $\mathrm{Na}^{+}$a los tres meses posteriores a la plantación, y caracteres de calidad de la fruta que fueron peso (g), longitud $(\mathrm{cm})$, diámetro $(\mathrm{cm})$, firmeza externa e interna $(\mathrm{N})$ y contenido de sólidos solubles $(\%)$ en los primeros tres cortes. La concentración de $\mathrm{NO}_{3}^{-}, \mathrm{K}^{+}$y Na${ }^{+}$se determinó en el extracto celular de peciolos mediante ionómetros específicos portátiles de Cardy-Horiba (Horiba Corporation, Japón), siguiendo las recomendaciones de Hochmuth (1994). Las lecturas del extracto se realizaron a las 10:00 am tomando los peciolos de cinco plantas/ unidad experimental por cada ion analizado. La relación de $\mathrm{K}^{+} / \mathrm{Na}^{+}$se estimó a partir de la concentración promedio de potasio y sodio. El peso se obtuvo del promedio de cinco frutos con la ayuda de una báscula digital. La longitud y el diámetro se midieron con una regla graduada de $30 \mathrm{~cm}$ y vernier, respectivamente. $\mathrm{La}$ firmeza se cuantificó con el medidor de fuerza digital Chatillon DFS II (AMETEK Inc., USA), realizando punciones al azar en tres partes del exocarpo del fruto para la firmeza externa y del mesocarpo para la firmeza interna. El contenido de sólidos solubles se determinó en el extracto de jugo de la pulpa con un refractómetro digital Atago (ATAGO Inc., USA.).

Los datos obtenidos se sometieron a un análisis de varianza y prueba de comparación de medias de t-Student $(\mathrm{P}<0,05)$ para pares de medias ajustadas con el procedimiento Modelo Lineal General (GLM), usando el paquete estadístico SAS versión 9,1. Para las variables donde la interacción no fue significativa, las comparaciones de medias se realizaron por la prueba de Tuckey $(\mathrm{P}<0,05)$.

\section{Resultados y discusiones Concentración de iones}

El contenido de potasio en la hoja de pepino fue variable por la condición de planta, así como por la combinación condición de planta y concentración de salinidad (Tabla 1). De manera general, la planta injertada superó en $52,6 \%$ a la condición normal. La mayor respuesta de $\mathrm{K}^{+}$ acumulado se presentó en la planta injertada a una CE de $3,0 \mathrm{dS} \mathrm{m}^{-1}$, superando en 2585 ppm a la sin injertar (Figura 1). Esto indica que la condición de la planta (normal o injertada), dependiendo del grado de salinidad, será el nivel de potasio en la hoja. Niveles superiores de $\mathrm{K}^{+}$en la planta injertada con relación a la planta normal indican que el

Tabla 1. Medias y significancia estadística de concentración de potasio $\left(\mathrm{K}^{+}\right)$, sodio $\left(\mathrm{Na}^{+}\right)$ y relación potasio-sodio $\left(\mathrm{K}^{+} / \mathrm{Na}^{+}\right)$en extracto peciolar de hoja en pepino cv. Tirano en condición normal e injertada bajo diferentes niveles de salinidad.

\begin{tabular}{lcccc}
\hline \multicolumn{2}{c}{ Factores y niveles de variación } & $\mathrm{K}^{+}(\mathrm{ppm})$ & $\mathrm{Na}^{+}(\mathrm{ppm})$ & $\mathrm{K}^{+} / \mathrm{Na}^{+}$ \\
\hline \multirow{2}{*}{ Salinidad (S) } & 3,00 & $3.152,56 \mathrm{a}$ & $2.460,03 \mathrm{~b}$ & $2,75 \mathrm{a}$ \\
& 4,50 & $3.042,71 \mathrm{a}$ & $3.083,06 \mathrm{~b}$ & $2,28 \mathrm{a}$ \\
& 6,00 & $2.751,60 \mathrm{a}$ & $5.771,20 \mathrm{a}$ & $0,61 \mathrm{~b}$ \\
\hline \multirow{2}{*}{ Condición de planta (C) } & Normal & $2.361,02 \mathrm{~b}$ & $5.685,30 \mathrm{a}$ & $0,46 \mathrm{~b}$ \\
& Injertada & $3.603,56 \mathrm{a}$ & $1.857,56 \mathrm{~b}$ & $3,30 \mathrm{a}$ \\
\hline $\mathrm{S}$ & & $\mathrm{ns}$ & $* * *$ & $* * *$ \\
$\mathrm{C}$ & & $* * *$ & $* * *$ & $* * *$ \\
$\mathrm{~S}$ x C & & $* *$ & $\mathrm{~ns}$ & $* *$ \\
\hline
\end{tabular}

Medias con letras iguales dentro de la misma columna dentro de cada factor de variación no son estadísticamente diferentes (Tukey $\mathrm{P}<0,05$ ). ns: no significativo, **: significancia al $1 \%$; ***: significancia al $0,1 \%$. 


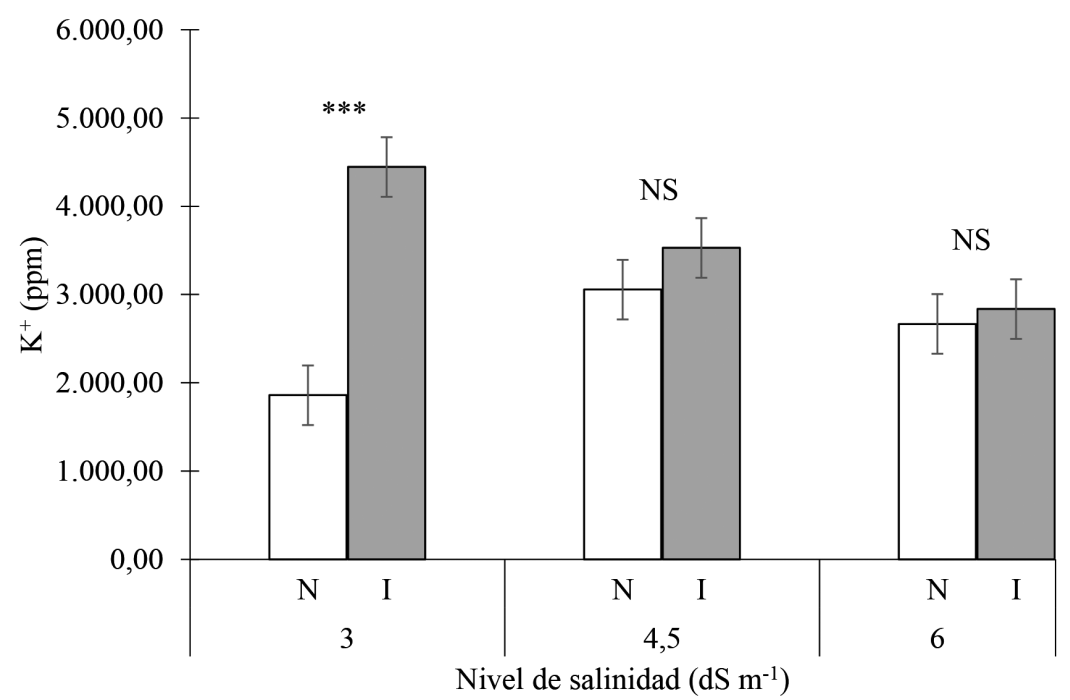

Figura 1. Contenido de potasio en savia de planta normal (N) e injertada (I) variedad Tirano en niveles de salinidad de 3,$0 ; 4,5$ y $6,0 \mathrm{dS} \mathrm{m}^{-1}$. Las medias con NS, y ***, dentro de cada nivel de salinidad son no significativas y $\mathrm{P} \leq 0,001$ (t-Student para pares de medias ajustadas).

portainjerto induce modificaciones en la planta, dependiendo del ambiente en que se desarrollen.

Respuestas similares fueron reportadas por Usanmaz y Abak (2019) al injertar pepino en el portainjerto híbrido (C. maxima x C. moschata) cv. Nun9075. Estos autores también señalan efectos de niveles bajos de $\mathrm{K}^{+}$en planta injertada al emplear los portainjertos híbridos RS841, TZ148 y Avar, en ambientes salinos de 5,0 y 7,5 $\mathrm{dS} \mathrm{m}^{-1}$. Los niveles bajos de $\mathrm{K}^{+}$en plantas de pepino son frecuentes debido a altas concentraciones de $\mathrm{Na}^{+}$, producto de la competencia entre $\mathrm{Na}^{+} \mathrm{y}$ $\mathrm{K}^{+}$por los sitios de unión en el transporte de $\mathrm{K}^{+}$ (Sonneveld y De Kreij, 1999). A nivel celular se induce el daño en la integridad de la membrana plasmática que lleva a la fuga de $\mathrm{K}^{+}$en la célula (Wang et al., 2013).

La concentración de sodio en savia de plantas de pepino fue modificada por la concentración de sales y la condición de la planta (Tabla 1). El contenido de sodio se incrementó en 25,3 y $134,6 \%$ al aumentar la conductividad eléctrica a 4,5 y $6,0 \mathrm{dS} \mathrm{m}^{-1}$, respectivamente. En cuanto a la condición de la planta, la concentración de sodio se redujo $67,3 \%$ en comparación con la condición normal. Colla y colaboradores (2013) reportaron una reducción de hasta $99,0 \%$ en el sodio acumulado en hoja de pepino injertado con el portainjerto Affyne (Cucumis sativus) a una concentración salina de $30 \mathrm{mM}$ de $\mathrm{NaCl}\left(6,1 \mathrm{dS} \mathrm{m}^{-1}\right)$.
Los mecanismos que inducen la tolerancia al estrés salino son la exclusión por la raíz o retención de $\mathrm{Na}^{+}$por el tejido del portainjerto y limitación del transporte de $\mathrm{Na}^{+}$al vástago (Edelstein et al., 2011). Sin embargo, las diferencias en las respuestas por efecto del injerto se deben a las variaciones genéticas de los portainjertos (Sykes, 2011). El uso del portainjerto híbrido $\mathrm{P} 360$ (C. máxima $\mathrm{x}$ C. moschata) en la variedad de pepino Ekron no redujo la acumulación de sodio en la hoja y tallo (Colla et al., 2013).

La relación del contenido de $\mathrm{K}^{+} / \mathrm{Na}^{+}$en la hoja fue modificada por efecto individual de los niveles de salinidad y la condición de planta, así como por la interacción de ambos factores en estudio (Tabla 1). La proporción de $\mathrm{K}^{+} / \mathrm{Na}^{+}$se redujo en 17,1 y $77,8 \%$ al aumentar el nivel de sales a 4,5 y $6,0 \mathrm{dS} \mathrm{m}^{-1}$, respectivamente. La planta injertada presentó la mejor relación de $\mathrm{K}^{+} / \mathrm{Na}^{+}$con un valor de 3,30 en comparación con la planta sin injertar $(0,4)$. En la interacción de ambos factores, la planta sin injertar mantuvo una relación muy baja $(0,5$ a 0,3$)$ al someterse a los distintos grados de salinidad (Figura 2). Las mayores proporciones significativas de potasio-sodio se obtuvieron en plantas injertadas desarrolladas a conductividades de 3,0 y 4,5 dS m-1 en comparación con la normal, con incrementos de 7,2 y 6,3 veces, respectivamente.

Usanmaz y Abak (2019) en trabajos similares encontraron niveles superiores de la relación 


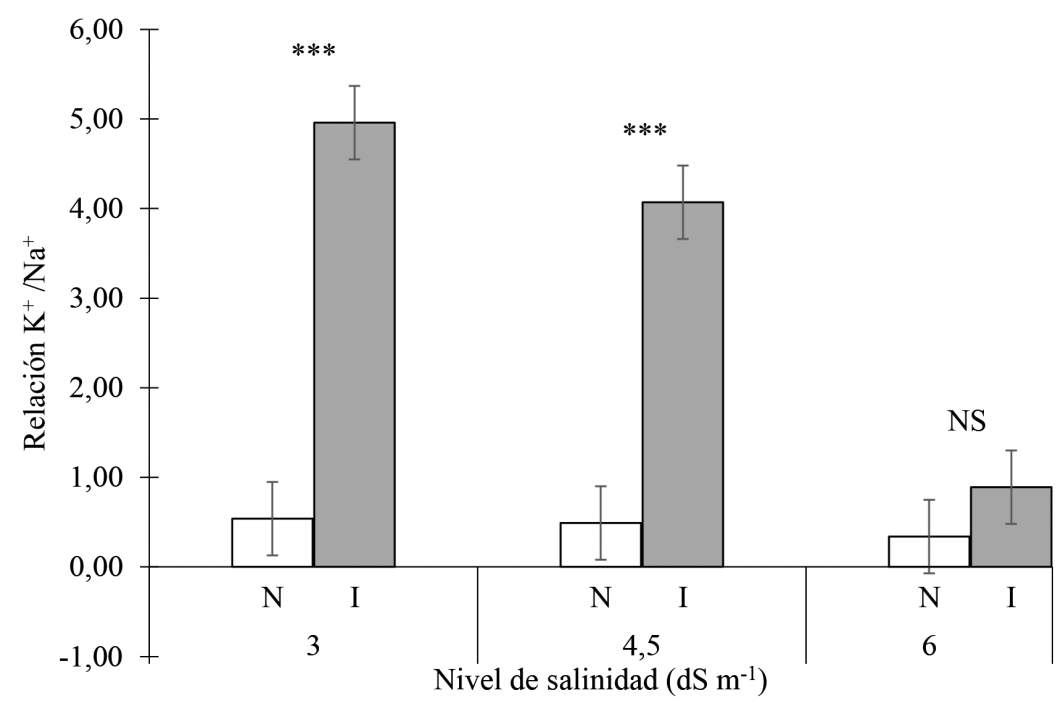

Figura 2. Relación potasio/sodio en savia de planta normal (N) e injertada (I) variedad Tirano en niveles de 3,0; 4,5 y $6,0 \mathrm{dS} \mathrm{m}^{-1}$ de salinidad. Las medias con NS y ***, entre grupos de salinidad son no significativas y $\mathrm{P} \leq 0,001$ (t-Student para pares de medias ajustadas).

de $\mathrm{K}^{+} / \mathrm{Na}^{+}$en plantas injertadas de pepino cv Falconstar con híbridos de calabaza (C. maxima x C. moschata) Nun905, RS841, TZ148 y Avar a diferentes concentraciones de salinidad. En plantas injertadas de pepino con el portainjerto de calabaza (C. moschata) se ha obtenido mayor proporción de $\mathrm{K}^{+} / \mathrm{Na}^{+}$en las hojas y el tallo del vástago al ser sometidos a una CE de $6,7 \mathrm{dS} \mathrm{m}^{-1}$ (Zhu et al., 2008). La mayor proporción de $\mathrm{K}^{+} / \mathrm{Na}^{+}$resulta de la capacidad selectiva de la planta para asimilar mayor cantidad de potasio y menor cantidad de sodio en condiciones salinas (Santa-Cruz et al., 2002). Mientras que la reducción en la relación $\mathrm{K}^{+} / \mathrm{Na}^{+}$tiene efecto negativo en la planta, ya que en el citosol activa las proteasas que inducen la muerte celular programada (Wang et al., 2013).

\section{Calidad de fruto}

El peso promedio de fruto varió por la concentración de salinidad, condición de la planta y por el efecto combinado de ambos factores (Tabla 2). El peso del fruto disminuyó 40,3 y 76,2 $\mathrm{g}$ al aumentar la conductividad a 4,5 y $6,0 \mathrm{dS} \mathrm{m}^{-1}$, respectivamente. Las plantas injertadas generaron frutos de mayor peso en comparación con las normales, con una superioridad de 39,8 g. El peso promedio de fruto fue mayor en planta injertada a un nivel de 3,0 y $6,0 \mathrm{dS} \mathrm{m}^{-1}$ en comparación con la condición normal, con aumento del 17,7 y $50,2 \%$, respectivamente (Figura 3 ). Resultados similares fueron reportados por Colla et al. (2012) al evaluar plantas de pepino, donde el peso de fruto fue de 234,0 $\mathrm{g}$ al emplear el portainjerto PS313 (C. maxima $\mathrm{x}$ C. moschata), mientras que en las plantas $\sin$ injerto el peso promedio fue $210,7 \mathrm{~g}$.

El aumento en peso se debe a la capacidad de las plantas injertadas para modificar aspectos morfológicos, fisiológicos y expresiones génicas a fin de mantener alta actividad fotosintética y estabilidad de la membrana, de tal manera que pueda tolerar la salinidad y mejorar los parámetros de crecimiento y rendimiento (Elsheery et al., 2020). No obstante, el comportamiento difiere por el tipo de portainjerto. $\mathrm{Al}$ utilizar las especies $C$. ficifolia y Lagenaria siceraria como portainjertos, estos no modificaron el peso del fruto con respecto a las plantas sin injertar dentro de los ambientes de $30 \mathrm{mM}$ y $60 \mathrm{Mm}$ de $\mathrm{NaCl}$ (Huang et al., 2009). Asimismo, un aumento en el peso se debe a una mayor dimensión (longitud y diámetro) del fruto aun en condiciones salinas (Suárez-Hernández et al., 2019).

La longitud de fruto no se modificó por el efecto individual o combinado de condición de la planta y grado de CE utilizado en los sustratos (Tabla 2), manteniendo un promedio entre 16,6 y $19,2 \mathrm{~cm}$. Resultados similares fueron obtenidos por Chao y Yen (2013), quienes no observaron 
Tabla 2. Medias y significancia estadística de peso (PF), longitud (LF), diámetro (DF), firmeza externa (FE), firmeza interna (FI) y contenido de sodios solubles (CSS) en frutos de pepino cv. Tirano en condición normal e injertada bajo diferentes niveles de salinidad.

\begin{tabular}{lccccccc}
\hline \multicolumn{2}{c}{ Factores y niveles de variación } & PF $(\mathrm{gr})$ & LF $(\mathrm{cm})$ & $\mathrm{DF}(\mathrm{mm})$ & FE $(\mathrm{N})$ & FI (N) & CSS (\%) \\
\hline \multirow{3}{*}{ Salinidad (S) } & 3,00 & $269,12^{\mathrm{a}}$ & $19,15^{\mathrm{a}}$ & $46,84^{\mathrm{a}}$ & $74,09^{\mathrm{a}}$ & $57,99^{\mathrm{a}}$ & $4,90^{\mathrm{a}}$ \\
& 4,50 & $228,82^{\mathrm{b}}$ & $19,14^{\mathrm{a}}$ & $44,93^{\mathrm{ab}}$ & $73,78^{\mathrm{a}}$ & $60,25^{\mathrm{a}}$ & $4,90^{\mathrm{a}}$ \\
& 6,00 & $192,90^{\mathrm{b}}$ & $16,63^{\mathrm{a}}$ & $42,14^{\mathrm{b}}$ & $74,09^{\mathrm{a}}$ & $60,95^{\mathrm{a}}$ & $5,07^{\mathrm{a}}$ \\
\hline \multirow{3}{*}{ Condición de planta (C) } & Normal & $210,39^{\mathrm{b}}$ & $17,37^{\mathrm{a}}$ & $42,79^{\mathrm{b}}$ & $73,85^{\mathrm{a}}$ & $61,86^{\mathrm{a}}$ & $5,00^{\mathrm{a}}$ \\
& Injertada & $250,18^{\mathrm{a}}$ & $19,24^{\mathrm{a}}$ & $46,48^{\mathrm{a}}$ & $74,12^{\mathrm{a}}$ & $74,12^{\mathrm{a}}$ & $4,91^{\mathrm{a}}$ \\
\hline S & & $* * *$ & NS & $* *$ & NS & NS & NS \\
C & & $* *$ & NS & $* *$ & NS & NS & NS \\
Cx S & & $*$ & NS & $* *$ & NS & NS & NS \\
\hline
\end{tabular}

Medias con letras iguales dentro de la misma columna dentro de cada factor de variación no son estadísticamente diferentes (Tukey P <0,05). NS: no significativo, *: significancia estadística al 5\%; **: significancia estadística al $1 \%$, ***: significancia estadística al $0,1 \%$.

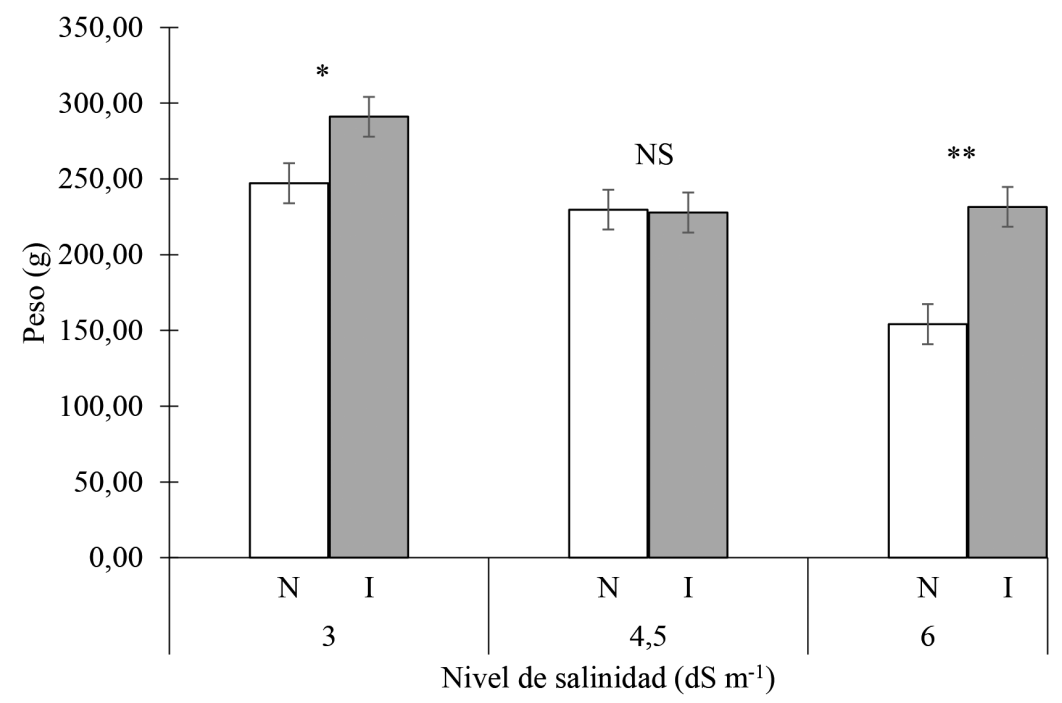

Figura 3. Peso de fruto de planta normal (N) e injertada (I) variedad Tirano en niveles de 3,0, 4,5 y $6,0 \mathrm{dS} \mathrm{m}^{-1}$ de salinidad. Las barras representan las medias ajustadas de la condición de la planta. Las medias con NS, * y **, entre grupos de salinidad por el contenido de potasio son no significativas, $\mathrm{P} \leq 0,05, \mathrm{P} \leq 0,01$ (t-Student para pares de medias ajustadas).

diferencias en la longitud de fruto de pepino al injertar sobre portainjertos Qingpi (Cucumis sp.) y Heroes (Cucurbita sp.).

El diámetro de fruto fue variable significativamente con la concentración salina y condición de planta, así como en la interacción de ambas (Tabla 2). El diámetro disminuyó por efecto de la salinidad en 4,1 y $10,0 \%$ al incrementar la concentración de sales a 4,5 y $6,0 \mathrm{dS} \mathrm{m}^{-1}$. De manera general, la planta injertada presentó un aumento de $3,7 \mathrm{~mm}$ en el diámetro del fruto en comparación con el obtenido de la planta normal. Los frutos más uniformes fueron los generados en las plantas injertadas en las distintas concentraciones evaluadas (Figura 4). El menor diámetro se registró en la planta normal a una CE de $6,0 \mathrm{dS} \mathrm{m}^{-1}$ con decremento del $17,7 \%$ con respecto al comportamiento a $3,0 \mathrm{dS} \mathrm{m}^{-1}$.

En otras investigaciones se ha encontrado que algunas especies de cucurbitáceas como $C$. 


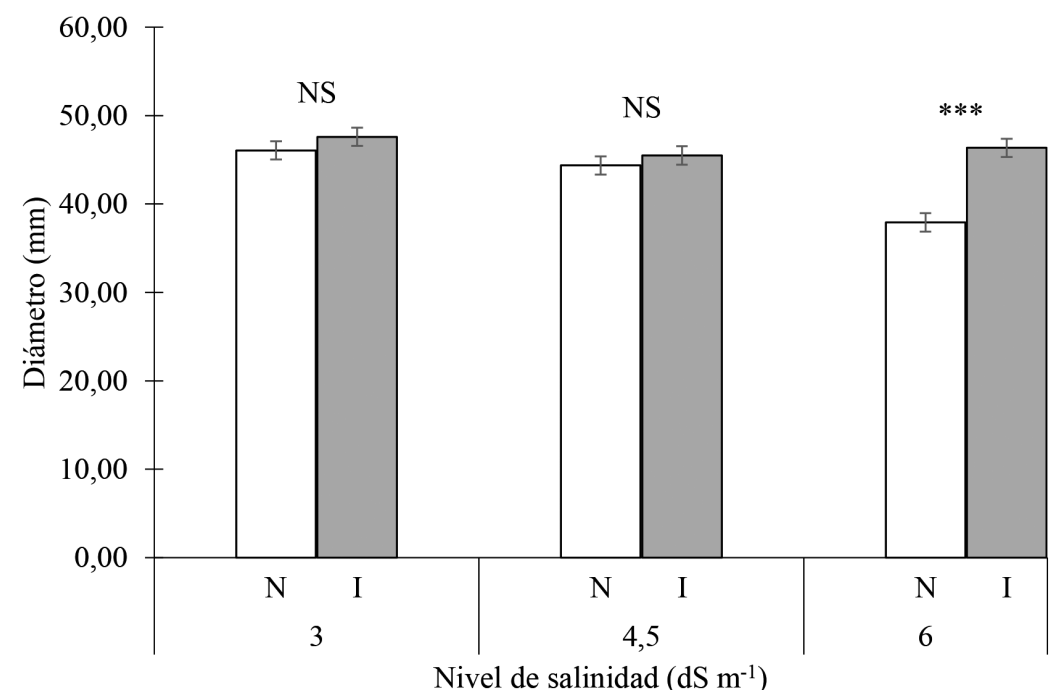

Figura 4. Diámetro de fruto de planta normal (N) e injertada (I) variedad Tirano en niveles de 3,0; 4,5 y 6,0 dS m-1 de salinidad. Las barras representan las medias ajustadas de la condición de la planta. Las medias con NS, y ***, entre grupos de salinidad por el contenido de potasio son no significativas, $\mathrm{P} \leq 0,05, \mathrm{P} \leq 0,01 \mathrm{y} \mathrm{P} \leq 0,001$ (t-Student para pares de medias ajustadas).

argyrosperma, C. ficifolia, Luffa cylindrica, y Cucumis $s p$. no modifican el diámetro del fruto al ser empleadas como portainjertos en pepino (Hernández-González et al., 2014; Chao y Yen, 2013). Esto fue evidente con nuestros resultados, donde se presentó una similitud del grosor de fruto en plantas injertadas y sin injertar al desarrollarse en ambientes menos estresantes de $\mathrm{NaCl}$ (CE de 3,0 y $4,5 \mathrm{dS} \mathrm{m}^{-1}$ ). Sin embargo, las dimensiones del tamaño del fruto pueden variar por el tipo de portainjerto y las condiciones en que se cultiven las plantas (Omar y El-hamahmy, 2019). Tal es el caso de nivel alto de $\mathrm{NaCl}\left(6,0 \mathrm{dS} \mathrm{m}^{-1}\right)$, donde el portainjerto Forticuke $\mathrm{F} 1$ presentó mayor grosor de fruto, relacionado posiblemente con una mayor asimilación de minerales como potasio y nitrato y menor absorción de sodio que facilita mayor tolerancia al estrés por $\mathrm{NaCl}$.

La textura (firmeza externa e interna) es una característica de los frutos de pepino que no fue modificada por efecto combinatorio o individual de condición de la planta y grado de salinidad sometido (Tabla 2). La firmeza externa del fruto de pepino en planta injertada y normal osciló entre 73,8 y 74,1 $\mathrm{N}$ en los distintos niveles de CE. La firmeza del mesocarpio (firmeza interna) registró valores similares entre 58,0 y 74,1 N independientemente de la condición de planta y grado de salinidad. Análisis sensoriales han demostrado que la textura en frutos no se modifica al emplear el portainjerto Carnivor (C. maxima $\mathrm{x}$ C. moschata) en las variedades de pepino Mirey F1 y Mina F1 (Velkov y Pevicharova, 2016). En frutos de sandía injertada con el portainjerto Strong Tosa (C. maxima $x$ C. moschata) se ha observado que la firmeza del mesocarpio se mantiene sin modificación en suelos altamente salinos (SuárezHernández et al. 2019).

El contenido de sólidos solubles es otro atributo de la fruta que no fue alterado de forma significativa por efecto individual o combinado de condición de la planta y grado de salinidad (Tabla 2). Los frutos de pepino presentaron valores similares entre 4,9 y $5,1 \%$ en plantas injertadas y sin injertar en los diferentes grados de salinidad. Respuestas similares fueron obtenidas al utilizar portainjertos Heroes (Cucurbita sp.), Affyne ( $C$. moschata) y P360 (C. maxima $x$ C. moschata) en plantas de pepino bajo condiciones de estrés por $\mathrm{NaCl}$, donde el contenido de sólidos solubles no fue modificado significativamente (Chao y Yen, 2013; Colla et al., 2013).

\section{Conclusión}

El uso del portainjerto híbrido Forticuke F1 (C. maxima x C. moschata) en la planta de pepino favorece la asimilación de potasio y mantiene 
alta relación de potasio/sodio a concentraciones salinas de 4,5 y $6,0 \mathrm{dS} \mathrm{m}^{-1}$. El transporte de sodio hacia las partes aéreas de pepino es limitado por el portainjerto híbrido en ambientes de 3,0; 4,5 y $6,0 \mathrm{dS} \mathrm{m}^{-1}$. La planta injertada incrementó el peso y diámetro de fruto en el ambiente de $6,0 \mathrm{dS} \mathrm{m}^{-1}$. La longitud, firmeza del epicarpio y mesocario, y el contenido de sólidos solubles no fueron modificados por la planta injertada en los niveles de salinidad de 3,$0 ; 4,5$ y $6,0 \mathrm{dS} \mathrm{m}^{-1}$.

\section{Literatura Citada}

Chao, H.F.; Yen, Y.F.

2013. Effects of Cucumis and Cucurbita Rootstocks on Vegetative Traits, Yield and Quality in 'Tainan $\mathrm{N}^{\circ} 1^{1}$ ' Cucumber. Journal of Horticultural Sciences, 8 (1): 51-54. Colla, G.; Rouphael, Y.; Jawad, R.; Kumar, P.; Rea, E.; Cardarelli, M.

2013. The effectiveness of grafting to improve $\mathrm{NaCl}$ and $\mathrm{CaCl} 2$ tolerance in cucumber. Scientia Horticulturae, 164: 380-391.

Colla, G.; Rouphael, Y.; Rea, E.; Cardarelli, M.

2012. Grafting cucumber plants enhance tolerance to sodium chloride and sulfate salinization. Scientia Horticulturae, 135: 177-185.

Edelstein, M.; Plaut, Z.; Ben-Hur, M.

2011. Sodium and chloride exclusion and retention by nongrafted and grafted melon and Cucurbita plants. Journal of Experimental Botany, 62 (1): 177-184.

Elsheery, N.I.; Helaly, M.N.; Omar, S.A.; John, S.V.S.;

Zabochnicka-Swiatek, M.; Kalaji, H.M.; Rastogi, A.

2020. Physiological and molecular mechanisms of salinity tolerance in grafted cucumber. South African Journal of Botany, 130: 90-102.

Grattan, S.R.; Grieve, C.M.

1999. Salinity-mineral nutrient relations in horticultural crops. Scientia Horticulturae, 78 (1-4): 127-157.

Heidari, A.A.; Kashi, A.; Saffari, Z.; Kalatejari, S.

2012. Effect of different Cucurbita rootstocks on survival rate, yield and quality of greenhouse cucumber cv. Khassib. Plant Ecophysiology, 2 (3): 115-120.

Hernández-González, Z.; Sahagún-Castellanos, J.; Espinosa-

Robles, P.; Colinas-León, M.T.; Rodríguez-Pérez, J.E.

2014. Efecto del patrón en el rendimiento y tamaño de fruto en pepino injertado. Revista Fitotecnia Mexicana, 37 (1): 41-47.

Hochmuth, G.

1994. Plant petiole sap-testing for vegetable. Cooperative Extension Service Circular No 1144. IFAS, USA. 5 p.

Huang, Y.; Bie, Z.; He, S.; Hua, B.; Zhen, A.; Liu, Z. 2010. Improving cucumber tolerance to major nutrients induced salinity by grafting onto Cucurbita ficifolia. Environmental and Experimental Botany, 69: 32-38.

Huang, Y.; Tang, R.; Cao, Q.; Bie, Z.

2009. Improving the fruit yield and quality of cucumber by grafting onto the salt tolerant rootstock under $\mathrm{NaCl}$ stress. Scientia Horticulturae, 122 (1): 26-31.

Maroto, J.V.; Borrego, I.; Miguel-Gómez, A.; Pomares-García, F. 2002. El cultivo de la sandía. Mundi-prensa, España. 322 p.

Mengel, K.; Kirkby, E.A.

2001. Principles of plant nutrition (5th ed). Springer, Netherlands. 848 p.
Mohsin, S.M.; Hasanuzzaman, M.; Bhuyan, M.H.M.B.; Parvin, K.; Fujita, M.

2019. Exogenous tebuconazole and trifloxystrobin regulates reactive oxygen species metabolism toward mitigating saltinduced damages in cucumber seedling. Plants, 8: 428.

Omar, G.F.; El-hamahmy, M.A.M.

2019. Effect of rootstocks on vegetative growth, yield and fruit quality of cucumber. Hortscience Journal of Suez Canal University, 8 (1): 1-10.

Pulido-Madrigal, L.

2016. Cambio climático, ensalitramiento de suelos y producción agrícola en áreas de riego. Terra Latinoamericana, 34 (2): 207-218.

Santa-Cruz, A.; Martínez-Rodríguez, M.M.; Pérez-Alfocea,

F.; Romero-Aranda, R.; Bolarin, M.C.

2002. The rootstock effect on the tomato salinity response depends on the shoot genotype. Plant Science, 162 (5): 825-831.

Sonneveld, C.; De Kreij, C.

1999. Response of cucumber (Cucumis sativus L.) to an unequal distribution of salts in the root environment. Plant Soil, 209 (1): 47-56.

Suárez-Hernández, A.M.; Vázquez-Angulo, J.C.; GrimaldoJuárez, O.; Ceceña-Durán, C.; González-Mendoza, D.; Bazante-González, I.; Mendoza-Gómez, A.

2019. Production and quality of grafted watermelon in saline soil. Horticultura Brasileira, 37 (3): 215-220.

Sykes, S.R.

2011. Chloride and sodium excluding capacities of citrus rootstock germplasm introduced to Australia from the People's Republic of China. Scientia Horticulturae, 128 (4): 443-449.

Usanmaz, S.; Abak, K.

2019. Plant growth and yield of cucumber plants grafted on different commercial and local rootstocks grown under salinity stress. Saudi Journal of Biological Sciences, 26 (6): 1134-1139.

Velkov, N.; Pevicharova, G.

2016. Effects of cucumber grafting on yield and fruit sensory characteristics. Zemdirbyste-Agriculture, 103 (4): 405-410.

Wan, S.; Kang, Y.; Wang, D.; Liu, S.

2010. Effect of saline water on cucumber (Cucumis sativus L.) yield and water use under drip irrigation in North China. Agricultural Water Management, 98 (1): 105-113.

Wang, M.; Zheng, Q.; Shen, Q.; Guo, S.

2013. The Critical Role of Potassium in Plant Stress Response. International Journal of Molecular Sciences, 14 (2): 7370-7390.

Zhu, J.; Bie, Z.; Huang, Y.; Han, X.

2008. Effects of grafting on the growth and ion concentrations of cucumber seedlings under $\mathrm{NaCl}$ stress. Soil Science and Plant Nutrition, 54 (6): 895-902. 